\title{
An integral-free expression for short-term changes in fault stability due to pore pressure induced when a point load is placed on the pervious boundary of a porous elastic half space containing a fault
}

\author{
RAMEsh Chander ${ }^{1}$ and S K TOMAR ${ }^{2, *}$ \\ \#290 Sector - 4, Mansa Devi Complex, Panchkula 134 114, Haryana, India. \\ ${ }^{2}$ Department of Mathematics, Panjab University, Chandigarh 160 014, India. \\ *Corresponding author. e-mail: sktomar66@gmail.com
}

A concentrated load with step-function time behaviour is placed normal to the planar, pervious boundary of a porous elastic half space (PEHS) with compressible constituents. A planar fault exists in the PEHS in such a way that the poroelastic behaviour of the medium is unhindered. We derive an approximate but integral-free expression for CFSCPP, i.e., changes in fault stability due to changes in pore pressure, at a point not too far off the line along which the load acts. But, in the interest of simplicity, the main discussion is focussed on a consideration of CFSCPP at a point $P$ located on the fault at depth $z$ directly beneath the load. It is convenient to introduce dimensionless time $t_{D}$ directly proportional to real time $t$. The constant of proportionality is $4 c / z^{2}$, where $c$ is hydraulic diffusivity. The derived approximate expression gives results with an accuracy of greater than $99 \%$ for limited values of $t_{D}$ after the load is imposed. We learn from the derived expression that, for a given $z$, fault stability undergoes an initial sudden decrease commensurate with the undrained pore pressure induced in the PEHS. This is followed by a more gradual decrease in fault stability with increasing $t_{D}$ until a minimum is reached. The real time $t$ to minimum fault stability increases with $z$. The magnitude of CFSCPP decreases with $z$ as $z^{-2}$ for a given $t_{D}$ in the permissible range. The derived expression and the inferences based on it should be useful during earth science investigations of the possible hazards due to reactivation of a pre-existing shallow fault when a civil engineering project involving imposition of a heavy load on the earth's surface is to be executed nearby. They should be useful also for investigations if a shallow earthquake occurs near such a project soon after its execution.

\section{Introduction}

Investigations of fault stability are important from the stand point of geological hazards, specifically the seismic hazard. A tectonic earthquake occurs every time a pre-existing fault gets reactivated in the earth. Of course, such an earthquake will occur also when a fresh fault is formed in rocks. We focus here on reactivation because so many faults have formed already at shallow depths in the earth over geologic time. Also, the conditions commonly considered for fault reactivation are slightly less severe than those for formation a fresh fault because the cohesive strength of the fault is usually small and is ignored (Sibson 1985). At the current stage of our knowledge, the criterion for initiation of fault

Keywords. Rock mechanics; geomechanics; poroelasticity; pore pressure; half space, point load; faults; fault stability; earthquakes; seismic hazard. 
slip through reactivation (e.g., Sibson 1985) is the same regardless of the magnitude of the accompanying earthquake. The criterion (Jaeger and Cook 1969; Sibson 1985) states that a pre-existing fault will be reactivated and will slip at a point when the shear stress acting there exceeds the frictional stress. The frictional stress is the product of the fault friction coefficient and the difference between the compressive normal stress and pore pressure acting at the point in the fault. It is recognized that the pore pressure and the shear and normal stresses may arise from many different causes of varying strengths.

We consider in this article not fault stability but only CFSCPP, i.e., changes in fault stability due to changes in pore pressure acting on the fault. The changes in fault stability due to changes in stresses acting on the fault are not taken up because that would have required an even more elaborate and extensive analysis than has been carried out here. Hubbert and Rubey (1959) highlighted the role of pore pressure in the initiation of fault slip. They were addressing an iconic problem of geology, namely, how to explain the horizontal movement of a large rock mass in the hanging wall of a low angle thrust fault over tens of kilometers, and sometimes even a few hundred kilometers (see also Price 1988). Investigations of the Denver earthquakes of the early 1960's, induced by injection of water in deep boreholes provided a rare, but full scale, in situ experiment to document the role of pore pressure in the occurrence of tectonic earthquakes (Healy et al. 1968). Interest in the influence of pore pressure on fault stability was intensified by Snow's (1972) rock mechanics-based analysis of earthquakes near new reservoirs.

Our interest here is in those instances of CFSCPP which arise due to loads applied more or less suddenly on the earth's surface due to activities of man. Examples of such loads are heavy civil engineering structures and reservoirs impounded behind dams. Any construction on a hill slope where a surface of slip exists from earlier landslides is also a close enough example. Our attention has been drawn in this regard to shifting of substantial loads at the earth's surface during mining operations. CFSCPP also would occur for loads placed on or removed from the earth's surface suddenly through geological processes such as mass movements in landslides. A load removal has the effect on CFSCPP opposite to that due to load imposition. Finally, on the basis of the principle of uniformitarianism, it is expected that CFSCPP would have occurred also in the geologic past under similar circumstances.

The CFSCPP due to causes just mentioned will be relatively small and it will not be sufficient to initiate slip on the fault by itself. But an increase in pore pressure will always lead to an increase in the tendency of the fault to slip and vice versa. It may happen sometimes that stresses and pore pressure acting on a fault due to ambient causes may have reached near critical levels for initiation of slip. The CFSCPP due to a load imposed on the surface may then be sufficient to permit slip to occur on the fault and thus to induce an earthquake. The possibility, however remote, that this hazard may materialise, justifies efforts to facilitate geological and geophysical investigations of pre-existing faults near sites of important civil engineering structures. The efforts may include theoretical studies, such as the present one, even if computer-based numerical procedures are in place or may be implemented. Theoretical studies frequently help us to rationalize and gain useful insights about the involved phenomena and their effects.

The theoretical analyses of Bell and Nur (1978) and Roeloffs (1988) also pertain to simulation of changes in fault stability, even though their final results are not expressed through integral-free formulas. The earth is modelled in both of those studies as a fully coupled porous elastic half space (PEHS) in plane strain deformation. The 'fully coupled' here means that changes in mean normal stress at a point in a PEHS induce changes in pore pressure and vice versa. The bounding surface of the PEHS in these analyses is acted upon by a load and a source of pore pressure that have an unrealistic infinite extent in one horizontal direction and a limited extent in the perpendicular horizontal direction. We consider instead the case of an axially symmetric point load on the surface of a fully coupled PEHS because an ensemble of point loads can represent a load of finite horizontal extent and arbitrary shape in map view with much greater fidelity. The case of a point source of pore pressure may be taken up in due course.

Boussinesq formulae (Jaeger and Cook 1969) for a point load imposed on an ideal elastic half space are adequate for initial analyses of fault stability in the dry state. Use of a PEHS model for simulation is a logical next step for systematic, simultaneous estimation of pore pressure and elastic stresses. It appears that such an analysis has been possible for nearly two decades (see Singh et al. 2009 and Wang 2000, p. 229 who cited article by Rajapakse and Senjuntichai 1993). In that article, the inverse Laplace transform for pore pressure was evaluated approximately and the inverse Hankel transform integral was evaluated numerically. We have been able to solve an equivalent inverse Fourier exponential transform for pore pressure exactly. We have sought to replace numerical evaluation of the inverse Hankel transform integral by an analytical result also. But to achieve this aim, we had to expand the integrand into multiple infinite 
series. The inverse Hankel transform of each term in each of those series can be evaluated analytically exact. Our result is approximate because we consider only one or two leading terms of each of those series. Still, in the space of independent variables involved, there is a domain in which the formula gives results to an accuracy of better than $99 \%$. Therefore, it is as good as an exact formula would have been in that domain.

The mathematical analysis at the core of this article has been placed in Appendix A to enhance readability of the main text. Two results, given in equations (A10) and (A11), are recalled and interpreted in the text below.

\section{Integral free expressions for CFSCPP}

Let us assume that a vertical concentrated load is imposed suddenly and then maintained forever on the otherwise free pervious surface of a fully coupled, homogeneous, isotropic PEHS. Let us also assume that a fault is embedded in this PEHS in such a way that the poroelastic processes are not affected. The orientation of the fault and the nature of relative slip between its two sides need not be specified because CFSCPP is the same in all cases. These data would have been critical if we had estimated simultaneously the changes in normal and shear stresses induced due to the surface point load.

For brevity and specificity in the following paragraphs, we identify points $P$ and $Q$ in the PEHS. Both points lie on the buried fault plane. But $P$ is located directly beneath the point load applied at the surface of PEHS. $Q$ is not too far from $P$.

We also recall that, when the cohesive strength is ignored, fault stability $S$, regardless of whether at $P$ or $Q$, is given (see, for example, Harris 1998, equation 1) by:

$$
S=(\sigma-p) \tan \phi-\tau .
$$

Here $\sigma$ is compressive normal stress and $\tau$ is the magnitude of the shear stress acting on the fault at $P$ or $Q ; p$ is pore pressure and $\tan \phi$ is the coefficient of friction for the fault in the PEHS. Then the contribution $\triangle S$ to change in fault stability due to change $\triangle p$ in pore pressure is given by

$$
\triangle S=-\tan \phi \triangle p
$$

An increase in pore pressure leads to decrease in fault stability.

\subsection{CFSCPP at P}

Equation (A10) of Appendix A is the most general result of this article as far as CFSCPP is concerned. It is applicable to both $P$ and $Q$. But we discuss here first, for convenience, the restricted result given in equation (A11) that is applicable only to $P$. Thus, CFSCPP at $P$ is

$$
\begin{aligned}
\triangle S\left(z, t_{D}\right) \approx & -\tan \phi\left(\frac{L}{2 \pi}\right)\left(\frac{1}{z^{2}}\right) \\
& \times\left[\left\{d_{1}+d_{2} \sqrt{t_{D}}-d_{3} t_{D}\right\}-d_{4} \frac{e^{-\frac{1}{t_{D}}}}{\sqrt{t_{D}}}\right] .
\end{aligned}
$$

In this equation, $z$ is the depth of $P$ beneath the surface of the PEHS and $L$ is the magnitude of the point load. Constants $d_{i}(i=1,2,3,4)$, depend on the properties of the PEHS. These constants are defined in Appendix A immediately after equation (A11). $t_{D}$ is dimensionless time. It is defined in terms of regular time $t$ through the relation $t_{D}=$ $4 c t / z^{2}, c$ being the hydraulic diffusivity of the PEHS. Regular time is to be measured from the instant when the load is imposed. If we wish to represent $\triangle S$ in terms of regular time then we merely have to replace $t_{D}$ in all four terms in the square brackets by using its defining relation. The advantage in using dimensionless time is that $z$ appears explicitly in equation (2) in only one factor, namely, $1 / z^{2}$.

The first three terms in the square brackets in equation (2) pertain to the effect on fault stability of instantaneous elastic stresses as they evolve with time in the PEHS under the applied load, and the fourth term reflects the effect on fault stability due to diffusion of water in the PEHS.

It is possible to use algebraic and calculus based procedures to deduce the physical implications of equation (2). But it is more convenient to achieve that aim by making this equation specific to a particular poroelastic material. Ruhr sandstone is one of the nine rocks for which Wang (2000) has provided numerical data about poroelastic properties. If these nine rocks are ranked according to the magnitudes of their hydraulic diffusivities, then Ruhr sandstone occupies one of the middle positions. According to Wang (2000), shear modulus of Ruhr sandstone is $13 \mathrm{GPa}$. Its Skempton coefficient is 0.88 , drained and undrained Poisson's ratios have values of 0.12 and 0.31 respectively, and hydraulic diffusivity is $0.0053 \mathrm{~m}^{2} \mathrm{~s}^{-1}$. We assume in addition that a friction coefficient of 0.6 is applicable to a fault in this rock. Ruhr sandstone has been considered in earlier studies of poroelasticity also (e.g., Singh et al. 2009). Thus $t_{D}=0.0212 t / z^{2}$ and equation (2) becomes:

$$
\begin{aligned}
\triangle S\left(z, t_{D}\right) \approx & -\frac{L}{z^{2}}\left[\left(0.0734+0.0358 \sqrt{t_{D}}-0.0270 t_{D}\right)\right. \\
& \left.-0.0828 e^{-1 / t_{D}} / \sqrt{t_{D}}\right] .
\end{aligned}
$$




\subsection{The domain of applicability of equation (2a)}

Since equation (2a) is approximate, we determine the domain of its applicability by comparing numerical values obtained from it with the exact values obtained by substituting the computer based evaluations of equation (A7), on right hand side of equation (1) above. Let $v_{a}$ and $v_{e}$ be the approximate and exact values of pore pressure for one specific set of numerical values for $z$ and $t_{D}$. Let $\left(v_{a}-v_{e}\right) 100 / v_{e}$ define the per cent error in $v_{a}$. Then, we would include this pair of $z$ and $t_{D}$ values in the domain of applicability of equation (2) if the accuracy of $v_{a}$ is better than $99 \%$. On the basis of a large number of calculations, we find this domain for Ruhr sandstone conservatively to be $0<z<\infty$, and $0 \leq t_{D}<0.2$.

We have verified that comparable results hold for other poroelastic materials in Wang's (2000) list.

\subsection{Time variation of CFSCPP at $P$}

Variation of CFSCPP over the permissible range of $t_{D}$ at $z=50 \mathrm{~m}$ is shown in figure 1. Exact results obtained from equation (A7) are included for comparison. The corresponding per cent errors in values obtained from equation (2a) are displayed in figure 2. Both the figures represent the effect of a point load of $10^{6} \mathrm{~N}$, which is the weight of water standing to a depth of $100 \mathrm{~m}$ over a horizontal area of $1 \mathrm{~m}^{2}$.

\section{General inferences about CFSCPP at $P$}

\subsection{CFSCPP at $P$ in the undrained state}

The undrained state in a poroelastic material refers to the situation in which the amount of water in

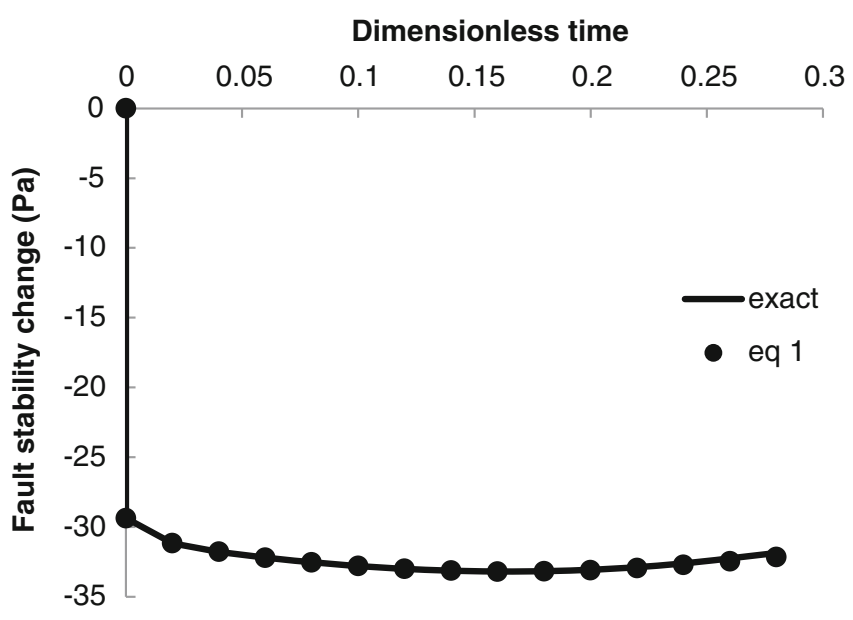

Figure 1. Comparison of CFSCPP results obtained from equation (1) with exact results from equation (A7) at a point at a depth of $50 \mathrm{~m}$ directly below the point load. The material composing the PEHS has properties of Ruhr sandstone. Point load has a magnitude of $10^{6} \mathrm{~N}$.

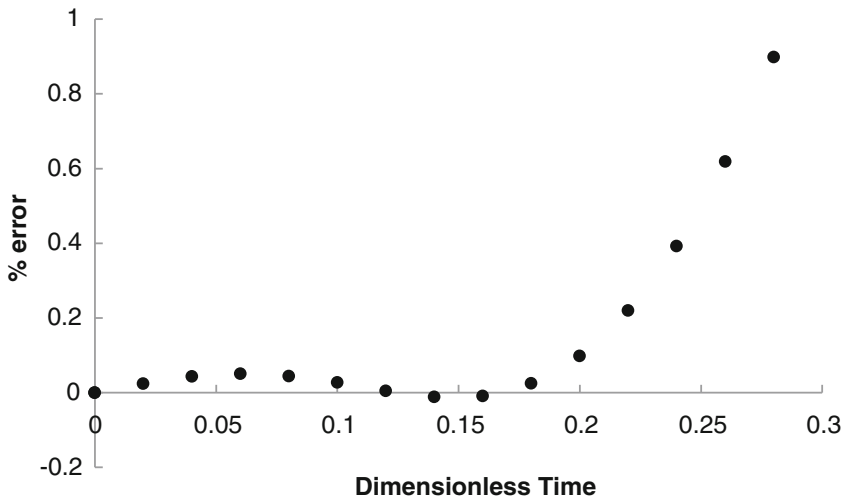

Figure 2. Percent error in results obtained from equation (1). This figure is based on the data used for figure 1 .

the pores has not had a chance to adjust to changes in the stress state. This state occurs in the present analysis at the exact instant of imposition of the load, i.e., at $t=t_{D}=0$. We learn from equation (2a) that

$$
\triangle S\left(z, t_{D}=0\right)=-0.0734 \frac{L}{z^{2}} .
$$

In other words, the undrained value of CFSCPP at $P$ in the PEHS with properties of Ruhr sandstone, as also for other poroelastic rocks, represents a sudden decrease in fault stability. The magnitude of CFSCPP at $P$ for the undrained condition decreases with $z$ as $z^{-2}$.

\subsection{Variation of CFSCPP at $P$ with increasing $t_{D}$}

The sudden decrease in fault stability at $P$ at the instant of imposition of the load is followed by a further gradual decrease with increasing $t_{D}$ until a minimum is reached (see figure 1). This holds for all values of $z$. The instant of the minimum in terms of regular time is different for different $z$. But in terms of $t_{D}$ the minimum occurs at 0.165 for all $z$. This value has been determined from the numerical results on which figure 1 is based. Similar behaviour is seen with other poroelastic materials mentioned by Wang (2000).

\subsubsection{The Mandel-Cryer effect}

Wang $(2000$, p. 150, 153-157) reviewed observational and theoretical work of Mandel and Cryer related to 'how poroelastic coupling can produce nonmonotonic pore-pressure behaviour following undrained loading (sic)'. The time dependent changes in fault stability deduced above are consistent with variation of pore pressure in the PEHS as identified in the Mandel-Cryer effect. 


\subsection{Rate of decrease of CFSCPP with $z$}

Equations (2) and (2a) imply that, for a given value of $t_{D}$ in the permissible range, the magnitude of CFSCPP also decreases with $z$ as $z^{-2}$. The constant of proportionality varies with the value of $t_{D}$

\section{CFSCPP at $Q$}

The case of CFSCPP at $Q$ is covered by equation (A10). Because of axial symmetry, greater generality is achieved by considering $Q$ on a conical surface whose apex is located at the point application of load and whose half apical angle is $\vartheta$. Equation (A11) and equations (2) and (2a) above correspond to the case $\vartheta=0$. Again there is a domain of applicability for which a limiting value of $\vartheta$ is specified also. Distance dependence is in terms of slant distance $R$ from the origin along the appropriate conical surface. Not surprisingly $R^{-2}$ variation is inferred, $z^{-2}$ being its special case for $\vartheta=0$. We also conclude that CFSCPP at $Q$ decreases suddenly to a value appropriate to the undrained pore pressure at $t=0$. It then decreases gradually until a minimum is reached.

\section{Discussion}

\subsection{Applicability of equation (2)}

Strictly speaking, equation (2) gives CFSCPP estimates at $P$ with an accuracy of $99 \%$ or better for dimensionless time up to a small value. Results of lesser accuracy would hold for larger values of this variable. This holds for all values of $z$.

\subsection{Applicability of the PEHS model}

A PEHS is an oversimplified model of the earth. The discrepancy between the model and the actual, heterogeneous, anisotropic situation in the earth, in general, increases as depth of the point on the fault under consideration increases. The effect of gravity and the internal heat of the earth are ignored in this model. Moreover, the PEHS model does not permit simulation of time-dependent rheological changes in the earth that may affect fault stability to some extent in rare cases. Lastly, use of the PEHS model for simulation of fault reactivation entails the assumption that, at the very least, a porous elastic material exists between the imposed load on the earth's surface and the point $P$ or $Q$ on the buried fault. Chances that this assumption holds will be more for a shallow fault rather than a deep fault in the earth. Geological and geophysical studies may be required to shed evidence in this regard in important cases.
There is another related constraint that is relevant to reactivation of deep faults near new reservoirs. Roeloffs (1988) found that a homogeneous PEHS model was adequate in rationalising the occurrence of post-impoundment earthquakes at $5 \mathrm{~km}$ depth under the manmade Lake Mead in USA. But our atention has been drawn to the possibility that, at such a depth, the pores in rocks may close under the prevailing overburden pressure. Other rock properties of interest too may change with increase in overburden pressure as well as increase in in situ temperature. The situation awaits further observations and analyses.

Meanwhile, the investigator will have to decide, on available evidence, the degree of confidence to be attached to the PEHS model and the related results based ultimately on equation (A10).

\section{Conclusion}

We have derived an integral-free formula to estimate CFSCPP that arise when a manmade load is imposed on the earth's surface. Due to the relative simplicity of the formula, we can draw several general conclusions from it about CFSCPP. Again, confining attention to point $P$ on the fault directly beneath the surface load, we learn that fault stability decreases suddenly by an amount commensurate with the undrained pore pressure induced on the imposition of the surface load. Fault stability at $P$ continues to decrease gradually with dimensionless time until a minimum is reached. We also learn that, at a given value of dimensionless time, the magnitude of CFSCPP varies as the inverse square of the depth of $P$. The derived formula and these inferences should be helpful during assessment of the possible hazard due to pore-pressure induced reactivation of a pre-existing shallow fault when a dam, a nuclear reactor, or a similarly important heavy civil engineering structure is to be constructed near it. Similarly, the present results should be helpful when the role of CFSCPP in the occurrence of a shallow earthquake near such a structure shortly after its completion is to be investigated.

\section{Acknowledgements}

Although no explicit citation is required, during the course of this research, we have benefitted from two internet sites, namely, integrals.wolfram.com and functions.wolfram.com. The helpful comments of the two referees, one of them in particular, are appreciated. 


\section{Appendix A}

\section{Mathematical derivation of an integral-free expression for CFSCPP}

\section{A1. Brief statement of the problem}

Continuing from the first two paragraphs of section 2 of the main text, let us assume a $(r, \theta, z)$ Cartesian cylindrical coordinate system whose origin lies in the boundary surface of the PEHS. The $z$-axis points into the PEHS. $G, \nu, \nu_{u}, B$ and $c$ (see Appendix B for definitions of symbols) are the requisite poroelastic constants. We wish to obtain an integral-free expression for CFSCPP in response to the imposed load.

The pore pressure considered in the following subsections A1.1-A3.3 of this appendix is actually the change $\triangle p$ in pore pressure induced by the imposed point load. However, the $\triangle$ is suppressed for convenience in these subsections.

We recall here briefly that McNamee and Gibson (1960) analysed the influence of surface loads on a PEHS conforming to Biot's model of poroelstic behaviour. But the PEHS material in their model had incompressible constituents. Roeloffs (1988) considered a PEHS with compressible constituents and full coupling between the developed stresses and pore pressure. Subsequently, progress has been made by considering a PEHS composed in part of finitely thick layers of porous elastic materials (e.g., Singh and Rani 2006). Singh et al. (2009) have presented expressions for stresses, displacements, and pore pressure in a PEHS with anisotropic permeability and compressible constituents subjected to an axially symmetric surface load.

\section{A1.1 Governing partial differential equations}

For the problem stated above, the following governing partial differential equations, expressed in terms of particle displacements and pore pressure, hold (Singh et al. 2009).

$$
\begin{gathered}
\frac{\partial^{2} u_{r}}{\partial r^{2}}+\frac{1}{r} \frac{\partial u_{r}}{\partial r}-\frac{u_{r}}{r^{2}}+\frac{\partial^{2} u_{r}}{\partial z^{2}}+\frac{1}{1-2 \nu} \frac{\partial \varepsilon}{\partial r}-\frac{\alpha}{G} \frac{\partial p}{\partial r}=0 \\
\frac{\partial^{2} u_{z}}{\partial r^{2}}+\frac{1}{r} \frac{\partial u_{z}}{\partial r}+\frac{\partial^{2} u_{z}}{\partial z^{2}}+\frac{1}{1-2 \nu} \frac{\partial \varepsilon}{\partial z}-\frac{\alpha}{G} \frac{\partial p}{\partial z}=0 \\
\eta \nabla^{2} p=G \nabla^{2} \varepsilon \\
\frac{\kappa}{\mu} \nabla^{2} p=\frac{\partial}{\partial t}\left(\alpha \varepsilon+\frac{p}{M}\right)
\end{gathered}
$$

Here,

$$
\begin{array}{r}
\varepsilon=\frac{\partial u_{r}}{\partial r}+\frac{u_{r}}{r}+\frac{\partial u_{z}}{\partial z}, \\
\nabla^{2}=\frac{\partial^{2}}{\partial r^{2}}+\frac{1}{r} \frac{\partial}{\partial r}+\frac{\partial^{2}}{\partial z^{2}} .
\end{array}
$$

The following are the constitutive equations applicable to the PEHS (Singh et al. 2009)

$$
\begin{aligned}
& \sigma_{z}=2 G\left(e_{z z}+\frac{\nu}{1-2 \nu} \epsilon\right)-\alpha p, \\
& \tau_{r z}=2 G e_{r z} .
\end{aligned}
$$

\section{A1.2 Boundary conditions}

The following conditions hold on the pervious boundary (at $z=0$ ) of the PEHS.

$$
\begin{aligned}
& p(r, 0, t)=0, \\
& \sigma_{z}(r, 0, t)=-L \delta(r) H(t), \\
& \tau_{r z}(r, 0, t)=0 .
\end{aligned}
$$

All field variables in the PEHS tend to 0 as $z$ tends to $\infty$.

\section{A1.3 Solution in the transform domain}

Singh et al. (2009) through equation (48) of their paper provide a formal expression for pore pressure in terms of inverse Laplace transform and zero order inverse Hankel transform integrals when a uniform load acts over a circular area of the PEHS. As suggested in that paper, that equation (48) may be modified using their equations (45) and (55) to deal with the case of a concentrated force on the surface of an isotropic PEHS. The modified equation (48) is our starting point for deriving an integral-free expression for pore pressure using analytical procedures exclusively. The symbol $s$ of Singh et al. (2009) is $\iota \omega$ for us. The angular frequency $\omega$ is the variable of integration of the inverse Fourier exponential transform (IFET) integral used by us in lieu of the inverse Laplace transform of that paper. Thus, we have:

$$
\begin{aligned}
& p(r, z, t)=\int_{0}^{\infty} \bar{p}(k, z, t) k J_{0}(k r) d k ; \\
& \bar{p}(k, z, t)=\frac{1}{2 \pi} \int_{-\infty}^{\infty} \overline{\bar{p}}(k, z, s) e^{s t} d \omega ;
\end{aligned}
$$


where

$$
\begin{aligned}
& \overline{\bar{p}}(k, z, s)=\left(\frac{1}{s}\right)\left(\frac{L}{2 \pi}\right)\left(\frac{\nu_{u}-\nu}{\eta}\right) \\
& \times \frac{\left(e^{-\frac{m z}{\sqrt{c}}}-e^{-k z}\right)\left(\frac{m}{\sqrt{c}}+k\right)}{\left[\left(\nu_{u}-\nu\right)\left(k-\frac{m}{\sqrt{c}}\right)-\left(1-\nu_{u}\right)\left(\frac{m}{\sqrt{c}}+k\right)\right]} .
\end{aligned}
$$

Further simplification of equation (A2) using equation (6) of Singh et al. (2009) yields

$$
\overline{\bar{p}}(k, z, s)=\left(\frac{L \gamma_{2}}{2 \pi}\right) \frac{\left(e^{-k z}-e^{-\frac{m z}{\sqrt{c}}}\right)}{s+2 \epsilon c k^{2}-2 \epsilon \sqrt{c} k m} .
$$

We wish to evaluate the integrals in equations (A1a) and (A1b) analytically using (A3).

\section{A2. Exact evaluation of the inverse Fourier exponential transform integral}

The radical associated with $m$ in the denominator of equation (A3) may be removed by rationalization, and the result may be expanded by the method of partial fractions to yield an eight term expression as:

$$
\begin{aligned}
\overline{\bar{p}}(k, z, s) & =\left(\frac{L \gamma_{2}}{2 \pi}\right)\left(\frac{2 \epsilon}{E}\right) \sum_{n=1}^{8} c_{n} F_{n}(s) \\
& =A_{1} B_{1} \sum_{n=1}^{8} c_{n} F_{n}(s) .
\end{aligned}
$$

The $c_{n}$ and $F_{n}(s),(n=1,2, \ldots, 8)$, are defined in table 1. The data from Campbell and Foster's (1948) tables that have been used to evaluate the following eight inverse Fourier exponential transform integrals (IFET) are also given in table 1. Inserting expression (A4) into equation (A1b), we have

$$
\bar{p}(k, z, t)=A_{1} B_{1} \sum_{n=1}^{8} c_{n}\left(\frac{1}{2 \pi}\right) \int_{-\infty}^{\infty} F_{n}(s) e^{s t} d \omega .
$$

The final result after substantial algebraic simplification is given by:

$$
\bar{p}(k, z, t)=\sum_{j=1}^{4} \bar{p}_{j}(k, z, t),
$$

where

$$
\begin{aligned}
\bar{p}_{1}(k, z, t)= & -A_{1} B_{1} e^{-k z} \operatorname{erfc}[\sqrt{c t} k], \\
\bar{p}_{2}(k, z, t)= & A_{1} B_{1} e^{-k z} \operatorname{erfc}\left[\sqrt{c t} k-\frac{z}{2 \sqrt{c t}}\right], \\
\bar{p}_{3}(k, z, t)= & A_{1} B_{1} \xi e^{-k z} \exp \left(-E c t k^{2}\right) \\
& \times \operatorname{erfc}[\xi \sqrt{c t} k], \\
\bar{p}_{4}(k, z, t)= & -A_{1} B_{1} \xi e^{\xi k z} \exp \left(-E c t k^{2}\right) \\
& \times \operatorname{erfc}\left[\xi \sqrt{c t} k+\frac{z}{2 \sqrt{c t}}\right] .
\end{aligned}
$$

Table 1. A short table of inverse Fourier exponential transform pairs extracted from Campbell and Foster (1948).

\begin{tabular}{lccl}
\hline$n$ & $c_{n}$ & $F_{n}(s)$ & \multicolumn{1}{c}{$f_{n}(t)=\frac{1}{2 \pi} \int_{-\infty}^{\infty} F_{n}(s) e^{s t} d \omega, t>0$} \\
\hline 1 & $e^{-k z}$ & $\frac{1}{s}$ & 1 \\
2 & $\xi e^{-k z}$ & $\frac{1}{s+E c k^{2}}$ & $e^{-E c t k^{2}}$ \\
3 & $\frac{m}{s}$ & $\frac{e^{-c t k^{2}}}{\sqrt{\pi} t}+\sqrt{c} k \operatorname{erf}(\sqrt{c t} k)$, \\
4 & $\frac{e^{-k z}}{\sqrt{c k}}$ & $\frac{m}{s+E c k^{2}}$ & $\frac{e^{-c t k^{2}}}{\sqrt{\pi t}}+\xi \sqrt{c} k e^{-E c t k^{2}} \operatorname{erf}(\xi \sqrt{c t} k)$ \\
5 & $-\frac{e^{-k z}}{\sqrt{c k}}$ & $\frac{e^{-m z / \sqrt{c}}}{s}$ & $e^{-k z}+\frac{1}{2}\left[e^{k z} \operatorname{erfc}\left(a^{+}\right)-e^{-k z} \operatorname{erfc}\left(a^{-}\right)\right]$ \\
6 & -1 & $\frac{e^{-m z / \sqrt{c}}}{s+E c k^{2}}$ & $\frac{e^{-E c t k^{2}}}{2}\left[e^{\xi k z} \operatorname{erfc}\left(b^{+}\right)+e^{-\xi k z} \operatorname{erfc}\left(b^{-}\right)\right]$ \\
7 & $-\xi$ & $\frac{m e^{-m z / \sqrt{c}}}{s}$ & $\sqrt{c k e^{-k z}}+\frac{e^{-c t k^{2}-\frac{z^{2}}{4 c t}}}{\sqrt{\pi t}}-\frac{\sqrt{c} k}{2}\left[e^{k z} \operatorname{erfc}\left(a^{+}\right)+e^{-k z} \operatorname{erfc}\left(a^{-}\right)\right]$ \\
8 & $-\frac{1}{\sqrt{c k}}$ & $\frac{m e^{-m z / \sqrt{c}}}{s+E c k^{2}}$ & $\frac{e^{-c t k^{2}-\frac{z^{2}}{4 c t}}}{\sqrt{\pi t}}-\frac{\xi \sqrt{c k} e^{-E c t k^{2}}}{2}\left[e^{\xi k z} \operatorname{erfc}\left(b^{+}\right)-e^{-\xi k z} \operatorname{erfc}\left(b^{-}\right)\right]$, \\
\hline
\end{tabular}

where $a^{ \pm}= \pm(z / 2 \sqrt{c t})+\sqrt{c t} k$ and $b^{ \pm}=(z / 2 \sqrt{c t}) \pm \xi \sqrt{c t} k$.

Data in columns 3 and 4 of row $3-8$ of this table pertain to transform pair nos. 548.1, 549, 818.1, 819, 821.1, 822 in Campbell and Foster (1948). 
All the IFET results listed by Campbell and Foster (1948) are analytically exact. We have made no approximations in proceeding analytically from equation (A3) to equations (A6a-d). Therefore, we suggest that the algebraic sum of analytical expressions in equations (A6a-d) constitute the exact IFET of the expression on the right hand side of equation (A3), when $t>0$.

\section{A3. Evaluation of the inverse Hankel transform integral}

\section{A3.1 The analytical and numerical procedures}

The single inverse Hankel transform (IHT) integral of equation (A1a) is replaced by four integrals using equations (A6-6d).

$$
\begin{aligned}
p(r, z, t) & =\sum_{j=1}^{4} p_{j}(r, z, t) \\
& =\sum_{j=1}^{4} \int_{0}^{\infty} \bar{p}_{j}(k, z, t) k J_{0}(k r) d k .
\end{aligned}
$$

These integrals may be evaluated numerically. But we show how they can be evaluated analytically using a series approach.

\section{A3.2 The infinite series and the retained terms}

The four complementary error functions in equations $(\mathrm{A} 6 \mathrm{a}-\mathrm{d})$ and one exponential in equation (A6c) are replaced by their series as given in equations (A8a), $(\mathrm{A} 8 \mathrm{c}),(\mathrm{A} 8 \mathrm{e})$ and $(\mathrm{A} 8 \mathrm{~g})$. The interlaced equations (A8b), (A8d), (A8f) and (A8h) indicate the terms retained from these series for further analysis.

$$
\begin{aligned}
& \bar{p}_{1}(k, z, t)= \\
& \quad-A_{1} B_{1} e^{-k z}\left[1-\frac{2}{\sqrt{\pi}} \sum_{n=0}^{\infty} \frac{(-1)^{n}(\sqrt{c t} k)^{2 n+1}}{n !(2 n+1)}\right],
\end{aligned}
$$

$$
\begin{aligned}
\bar{p}_{1 r}(k, z, \tau)= & -A_{1} B_{1} e^{-k z}\left[1-\frac{2 \sqrt{c t} k}{\sqrt{\pi}}\right], \\
\bar{p}_{2}(k, z, t)= & A_{1} B_{1} e^{-k z}\left[2-\frac{1}{\sqrt{\pi}} e^{-a^{2}}\right. \\
& \left.\times\left\{\frac{1}{a}+\sum_{n=1}^{\infty} \frac{1.3 \ldots(2 n-1)}{2^{n} a^{2 n+1}}\right\}\right],
\end{aligned}
$$

$$
\bar{p}_{2 r}(k, z, \tau)=A_{1} B_{1} e^{-k z}\left[2-\frac{1}{\sqrt{\pi}} e^{-a^{2}} \frac{2 \sqrt{c t}}{z}\right],
$$

$$
\begin{aligned}
\bar{p}_{3}(k, z, t)= & A_{1} B_{1} \xi e^{-k z}\left[\sum_{n=0}^{\infty} \frac{\left(-E c t k^{2}\right)^{n}}{n !}\right] \\
& \times\left[1-\frac{2}{\sqrt{\pi}} \sum_{n=0}^{\infty} \frac{(-1)^{n}(\xi \sqrt{c t} k)^{2 n+1}}{n !(2 n+1)}\right],
\end{aligned}
$$

$$
\bar{p}_{3 r}(k, z, \tau)=A_{1} B_{1} \xi e^{-k z}\left[1-\frac{2 \xi \sqrt{c t} k}{\sqrt{\pi}}\right]
$$

$$
\begin{aligned}
\bar{p}_{4}(k, z, t)= & -A_{1} B_{1} \xi e^{\xi k z} e^{-\left(E c t k^{2}\right)} \frac{1}{\sqrt{\pi}} e^{-b^{2}} \\
& \times\left[\frac{1}{b}+\sum_{n=1}^{\infty} \frac{1.3 \ldots(2 n-1)}{2^{n} b(2 n+1)}\right], \\
\bar{p}_{4 r}(k, z, \tau)= & -A_{1} B_{1} \xi e^{\xi k z} e^{-\left(E c t k^{2}\right)} \frac{1}{\sqrt{\pi}} e^{-b^{2}} \frac{2 \sqrt{c t}}{z} .
\end{aligned}
$$

The series in each of equations (A8a) and (A8e) is based on a definition of the error function (Abramowitz and Stegun 1972, 7.1.5). The series in each of equations (A8c) and (A8g) represents the asymptotic approximation for the complementary error function (Abramowitz and Stegun 1972, 7.1.24) because the quantities $a$ and $b$ in their arguments have large magnitudes for small $t$. The two series in equation (A8e) are multiplied before proceeding further. Finally, the $2 \sqrt{c t} / z$ in equations $(\mathrm{A} 8 \mathrm{~d})$ and $(\mathrm{A} 8 \mathrm{~h})$ represent the first term in the binomial series for $1 / a$ and $1 / b$ in equations (A8c) and $(\mathrm{A} 8 \mathrm{~g})$ respectively. The results in equations $(\mathrm{A} 8 \mathrm{~b}),(\mathrm{A} 8 \mathrm{~d}),(\mathrm{A} 8 \mathrm{f})$ and $(\mathrm{A} 8 \mathrm{~h})$ are approximate only because they represent a few terms retained from respective infinite series.

\section{A3.3 The derived expression for pore pressure}

The retained terms in equations (A8b), (A8d), (A8f) and (A8h) are substituted in equation (A7). Thus,

$$
\begin{aligned}
p_{r}(k, z, t)= & \int_{0}^{\infty} A_{1}\left[e^{-k z}\left\{1+\frac{4 \epsilon \sqrt{c t} k}{\sqrt{\pi}}-2 \epsilon \xi c t k^{2}\right\}\right. \\
& -\frac{2 \sqrt{c t}}{\sqrt{\pi} z} e^{\left.-\frac{z^{2}}{4 c t}-c t k^{2}\right]} k J_{0}(k r) d k .
\end{aligned}
$$

The integral may be evaluated term by term using the results listed at No. 20 on page 9 and No. 10 
on page 20 of Erdelyi et al. (1954, Vol. 2). We find that with $t_{D}=4 c t / R^{2}$

$$
\begin{aligned}
p\left(r, z, t_{D}\right)= & -\left(\frac{L \gamma_{2}}{2 \pi}\right)\left[\frac { z } { R ^ { 3 } } \left\{1+\frac{2 \epsilon t_{D}}{\sqrt{\pi}}\left(\frac{2 z^{2}-r^{2}}{z R}\right)\right.\right. \\
& \left.\left.-0.5 \epsilon \xi t_{D}\left(\frac{6 z^{2}-9 r^{2}}{R^{2}}\right)\right\}-\frac{2 e^{-\frac{1}{t_{D}}}}{\sqrt{\pi} z R \sqrt{t_{D}}}\right] .
\end{aligned}
$$

This equation may be written equivalently in terms of $R$ and $\vartheta$ using the relations $r=R \sin \vartheta, z=$ $R \cos \vartheta$. Thus,

$$
\begin{aligned}
& p\left(R, \vartheta, t_{D}\right) \approx \\
& \left(\frac{L \gamma_{2}}{2 \pi}\right)\left(\frac{1}{R^{2}}\right)\left[\operatorname { c o s } \vartheta \left\{1+\frac{2 \epsilon \sqrt{t_{D}}}{\sqrt{\pi}} \frac{\left(3 \cos ^{2} \vartheta-1\right)}{2 \cos \vartheta}\right.\right. \\
& \left.\left.-0.5 \epsilon \xi t_{D}\left(15 \cos ^{2} \vartheta-9\right)\right\}-\frac{2 e^{-\frac{1}{t_{D}}}}{\sqrt{\pi} \sqrt{t_{D}} \cos \vartheta}\right] . \text { (A } 9
\end{aligned}
$$

\section{A4. Change in fault stability}

The $p$ from equation (A9) is to be substituted in equation (1) of main text in place of $\triangle p$ to estimate CFSCPP at $Q$. Thus,

$$
\begin{aligned}
& \triangle S\left(R, \vartheta, t_{D}\right)=-\tan \phi p\left(R, \vartheta, t_{D}\right) \\
& \approx-\tan \phi \frac{L \gamma_{2}}{2 \pi} \frac{1}{R^{2}}\left[\operatorname { c o s } \vartheta \left\{1+\frac{2 \epsilon \sqrt{t_{D}}}{\sqrt{\pi}} \frac{\left(3 \cos ^{2} \vartheta-1\right)}{2 \cos \vartheta}\right.\right. \\
& \left.\left.-0.5 \epsilon \xi t_{D}\left(15 \cos ^{2} \vartheta-9\right)\right\}-\frac{2 e^{-\frac{1}{t_{D}}}}{\sqrt{\pi} \sqrt{t_{D}} \cos \vartheta}\right] \cdot(\mathrm{A} 10)
\end{aligned}
$$

Equation (A10) simplifies for the case of $P(\vartheta=0)$ to yield

$$
\begin{aligned}
\triangle S(z, \tau) \approx & -\tan \phi \frac{L}{2 \pi} \frac{1}{z^{2}}\left[\left\{d_{1}+d_{2} \sqrt{t_{D}}\right.\right. \\
& \left.\left.-d_{3} t_{D}\right\}-d_{4} \frac{e^{-\frac{1}{t_{D}}}}{\sqrt{t_{D}}}\right] .
\end{aligned}
$$

Here, $d_{1}=\gamma_{2}, \quad d_{2}=4 \gamma_{2} \epsilon / \sqrt{\pi}, \quad d_{3}=3 \gamma_{2} \epsilon \xi, \quad d_{4}=$ $2 \gamma_{2} / \sqrt{\pi}$.

Finally, we substitute values of poroelastic constants and coefficient of friction appropriate for Ruhr sandstone. We thus obtain an expression, specific to this rock, for CFSCPP at $P$.

$$
\begin{aligned}
\triangle S\left(z, t_{D}\right) \approx & -\frac{L}{z^{2}}\left[\left(0.0734+0.0358 \sqrt{t_{D}}\right.\right. \\
& \left.\left.-0.0270 t_{D}\right)-0.0828 \frac{e^{-\frac{1}{t_{D}}}}{\sqrt{t_{D}}}\right] .
\end{aligned}
$$

\section{Appendix B}

\section{List of symbols}

$a=\left(\frac{z}{2 \sqrt{c t}}-\sqrt{c t} k\right)$.
$b=\left(\frac{z}{2 \sqrt{c t}}+\xi \sqrt{c t} k\right)$.

$e_{i j}=$ strain components

erfc $=$ complementary error function

$k=$ wavenumber

$m=\sqrt{s+c k^{2}}$

$p=$ pore pressure

$r=$ radial space coordinate. It is also used as a subscript to indicate contribution due to retained terms

$s=\iota \omega$

$t=$ time

$t_{D}=\frac{4 c t}{z^{2}}=$ dimensionless time when considering variations with depth directly beneath the load

$u_{r}, u_{z}=r$ and $z$ components of particle displacement

$z=$ depth coordinate

$A_{1}=\frac{L \gamma_{2}}{2 \pi}$

$B_{1}=\frac{2 \epsilon}{E}$

$B=$ Skempton's coefficient (Wang 2000, p. 20)

$E=4 \epsilon(1-\varepsilon)$.

$G=$ Shear modulus

$H(t)=$ Heaviside unit step function

$R=\sqrt{r^{2}+z^{2}}$

$\alpha=$ Biot-Willis coefficient (Wang 2000, p. 21)

$M=$ Biot modulus, reciprocal of constant strain storage coefficient (Wang 2000, p. 20)

$\gamma_{2}=\frac{2}{3} B\left(1+\nu_{u}\right)=2 \mathrm{D}$ plane strain loading coefficient (Wang 2000, p. 160)

$\epsilon=\frac{\left(\nu_{u}-\nu\right)}{(1-\nu)}$

$\varepsilon=$ volumetric strain

$\theta=$ one of the cylindrical polar coordinates 
$\vartheta=$ angle between $R$ and the $z$-axis

$\kappa=$ intrinsic permeability of poroelastic medium

(Wang 2000, p. 81)

$\mu=$ fluid viscosity (Wang 2000, p. 81)

$\xi=1-2 \epsilon=\frac{\left(1+\nu-2 \nu_{u}\right)}{(1-\nu)}$. Note that $1+\xi=E / 2 \epsilon$

$\sigma_{i j}=$ stress components

$\nu_{u}=$ undrained Poisson's ratio

$\nu=$ Poisson's ratio

$\omega=$ angular frequency

$\phi=$ angle of friction

\section{References}

Abramowitz M and Stegun I A 1972 Handbook of Mathematical Functions with Formulas, Graphs, and Mathematical Tables; New York: Dover Publications, http:// people.math.sfu.ca/cbm/aands/frameindex.htm.

Bell Lee M and Nur A 1978 Strength changes due to reservoir induced pore pressure and stresses and application to Lake Oroville; J. Geophys. Res. 83(B9) 44694483.

Campbell G and Foster R 1948 Fourier Integrals for Practical Applications; New York: D Van Nostrand Company Inc.
Erdelyi A, Magnus W, Oberhettinger F and Tricomi F G 1954 Tables of Integral Transforms; Vol. 1 \& 2, McGrawHill, New York, Toronto and London.

Harris R 1998 Introduction to special section: Stress triggers, stress shadows and implications for seismic hazard; J. Geophys. Res.: Solid Earth 103 24,347-24,358.

Healy J H, Rubey W W, Griggs D T and Raleigh C B 1968 The Denver earthquakes; Science 161 1301-1310.

Hubbert M K and Rubey W W 1959 Role of fluid pressure in the mechanics of overthrust faulting; Bull. Geol. Soc. Am. 70 115-205.

Jaeger J C and Cook N G W 1969 Fundamentals of Rock Mechanics, Methuen, London, 515p.

McNamee J and Gibson R E 1960 Displacement functions and linear transforms applied to diffusion through porous elastic media; Q. J. Mech. Appl. Math. 13 99-111.

Price R A 1988 The mechanical paradox of large overthrusts; Geol. Soc. Am. Bull. 1000 1898-1908.

Roeloffs E A 1988 Fault stability changes induced beneath a reservoir with cyclic variations in water level; J. Geophys. Res.: Solid Earth 93(B3) 2107-2124.

Sibson R H 1985 A note on fault reactivation; J. Struct. Geol. 7 751-754.

Singh S J and Rani S 2006 Plane strain deformation of a multilayered poreolastic half-space by surface loads; J. Earth Syst. Sci. 115 685-694.

Singh S J, Kumar R and Rani S 2009 Consolidation of a poroelastic half-space with anisotropic permeability and compressible constituents by axially symmetric loading; J. Earth Syst. Sci. 118 563-574.

Snow D T 1972 Geodynamics of seismic reservoirs; Proceedings Symposium on Percolation through fissured rocks, German Society for Soil and Rock Mechanics, Stuttgart, T2J, pp. 1-9.

Wang H F 2000 Theory of Linear Poroelasticity with Applications to Geomechanics and Hydrogeology, Princeton University Press, Princeton. 